\title{
Characteristics of the Spanish neutron user community explored trough a bibliometric study
}

Scientific research using neutron scattering techniques began relatively recently in Spain. The first Spanish scientists to begin experiments did so in the 1970s. Initially, the neutron scattering experiments were reduced to topics related with materials science and/or magnetism, and only few researchers belonging to two or three different groups were involved in them. However, the number of researchers and research groups over the last 20 years, as well as the scientific disciplines to which they belong (physics, chemistry, engineering, geology, biology, medicine and even archaeometry and cultural heritage) have grown significantly.

Public institutions, in particular the government ministries responsible for research and science at various times, have been sensitive to the emergence and the rapid developing of this scientific discipline in Spain. Therefore, funding through research projects related to science using neutron scattering techniques has experimented a continuous increase in recent years. Moreover, Spain has also been involved in the international neutron source (Institut Laue Langevin (ILL), Grenoble, France), as an "associated scientific" country, for more than 20 years, being the first country, apart from the three founding countries (France, the United Kingdom and Germany), to become a partner of this major facility. More recently, Spain has also begun to participate in a similar way in the British spallation source (ISIS, Didcot).

Besides that, we would like to stress that most of the Spanish neutron users are members of the Spanish Society for Neutron Techniques (Sociedad Española de Técnicas Neutrónicas, SETN), a society with more than 250 members. The SETN is also one of the national associations shared into the European Neutron Scattering Association (ENSA). ${ }^{1}$

This contribution tries to summarize a more extended and detailed document, edited recently by the SETN, devoted to the evolution of the Spanish neutron scattering community since the early beginning, through the scientific output. ${ }^{2}$ The work is based on the bibliometric study of all the publications included in the international database Web of Science (WoS) in the period 19702006, making a particular emphasis on aspects related with collaboration between different research groups in Spain, and between them with groups in other countries.

Several questions are answered with this study, such as: how has the discipline been developed in recent years, in terms of the number of scientists, number of publications, and impact? Do the centres of activity increased in number? Is there a clear expansion of the research topics and the scientific areas covered by neutron techniques? Does the research using neutron scattering techniques have the characteristics of a big science? Which is the role that the Spanish scientific community play nowadays in the international context of this discipline?

Spanish scientific output on neutron techniques was obtained by identifying all documents in the WoS which featured any term derived from the lexeme neutron in the fields title, keywords or abstract, and the word Spain in the field affiliation. Scientific output by Spanish researchers from institutions abroad was also taken into account. Searches were limited to documents published between 1970 and 2006. After documents were downloaded, they were reviewed in order to refine the search and rule out those that were irrelevant to the discipline being analysed.

\footnotetext{
${ }^{1}$ For more details on the history of research into neutron techniques in Spain, please consult the Spanish Journal of Physics (of the Royal Spanish Society for Physics, [Real Sociedad Española de Física, RSEF]), Vol. 22, issue 4, pp. 2131 (2008).

2 "Thirty years of Spanish scientific research using neutron techniques: a well-established discipline"; Edt J. Campo and P. Gorria, ISBN: 13- 978-84-692-1286-8. Available at www.icmab.es/setn
} 
The study evaluates quantitative and semi-qualitative indicators based on international scientific publications, with the aim of obtaining a comprehensive overview of the development of Spanish research with neutron techniques over the last 30 years.

In order to study the 'big science' characteristics of the discipline and the degree of integration of the Spanish researchers into the international scientific community, the collaboration inside the area, particularly collaboration with other countries, and the presence of Spanish researchers in institutions abroad were studied.

The following criterion was adopted to quantify the size of the scientific community (in this case, the Spanish community which uses neutron techniques), and which can be, in our opinion, be used to evaluate any scientific discipline: "A frequent researcher in the field is one who has participated as co-author in at least 5 documents during last 15 years, and identified according to the selection criteria stated above." This criterion allows us to compare the sizes of a given scientific community in different countries objectively, and at the same time, to analyse their development over time.

Several findings indicate that research into neutron techniques in Spain is well established, has reached a maturity or consolidated age and shows a continuous growing tendency (see figure): A brief summary of the conclusions drawn from this study is given below

- Major increase in the number of documents, particularly from the beginning of the 1990s onwards. More than 2000 scientific articles related to neutron scattering techniques have been published in the period 1970-2006. This increase is much higher than that of the Spanish scientific output compiled as a whole along the same period. The "take-off" of the Spanish output occurs after 1987, when Spain became an associated scientific member of the Institut Max von Laue - Paul Langevin (ILL), an international point of reference in research using neutron techniques, and after the creation of the Spanish Society for Neutron Techniques, which currently has more than 250 members.

- Increased output is significantly linked to the increase in scientific collaboration in the field. National collaboration increases, contributing to the creation of networks within Spain, and especially to international collaboration, which is present in more than $70 \%$ of the articles. This facilitates the integration of Spanish researchers into the international scientific community.

- Growth in the expected and observed impact of output, which tends to be published in higher-prestige journals, and to receive more citations, over time.

- An increase in the diversification of research subjects over time. This favours the use of neutron scattering techniques in very different disciplines and with a large number of applications.

- An increase in the number of 'frequent scientists' both in Spain and abroad. This is even higher than the relative increase in the number of publications.

- Output and impact data for Spain can be placed in the global context. From the beginning of the 1980s onwards, Spain is climbing along the ranking of countries with most output, and now ranks 8th. The mean impact factor of the Spanish publications is above that of the worldwide average in the field.

Scientists belonging to research centres in France are present in more than half of Spanish publications with international collaboration, hence, this country is the more frequent collaborator of Spain. The latter is due to the neutron facilities located there, and in particular the ILL. A study of scientific collaboration networks of Spanish scientists in the field shows that they are clearly centred around the ILL, indicating particular dependency on the ILL for 
research. Over the years, new institutions have been incorporated into the network, increasing its size and the links between Spanish institutions, but the network remains centred around the ILL, which is its core.

In summary, the evolution of the Spanish research using neutron scattering techniques is closely linked to the increasing access of the Spanish researchers to neutron sources (in various countries around the world) which, are the nuclei of research in the area. The new European Spallation Source (ESS) will undoubtedly stimulate both competitive and high-quality scientific and technological research, guarantying also the needed training for young scientists and technology specialists in the field.

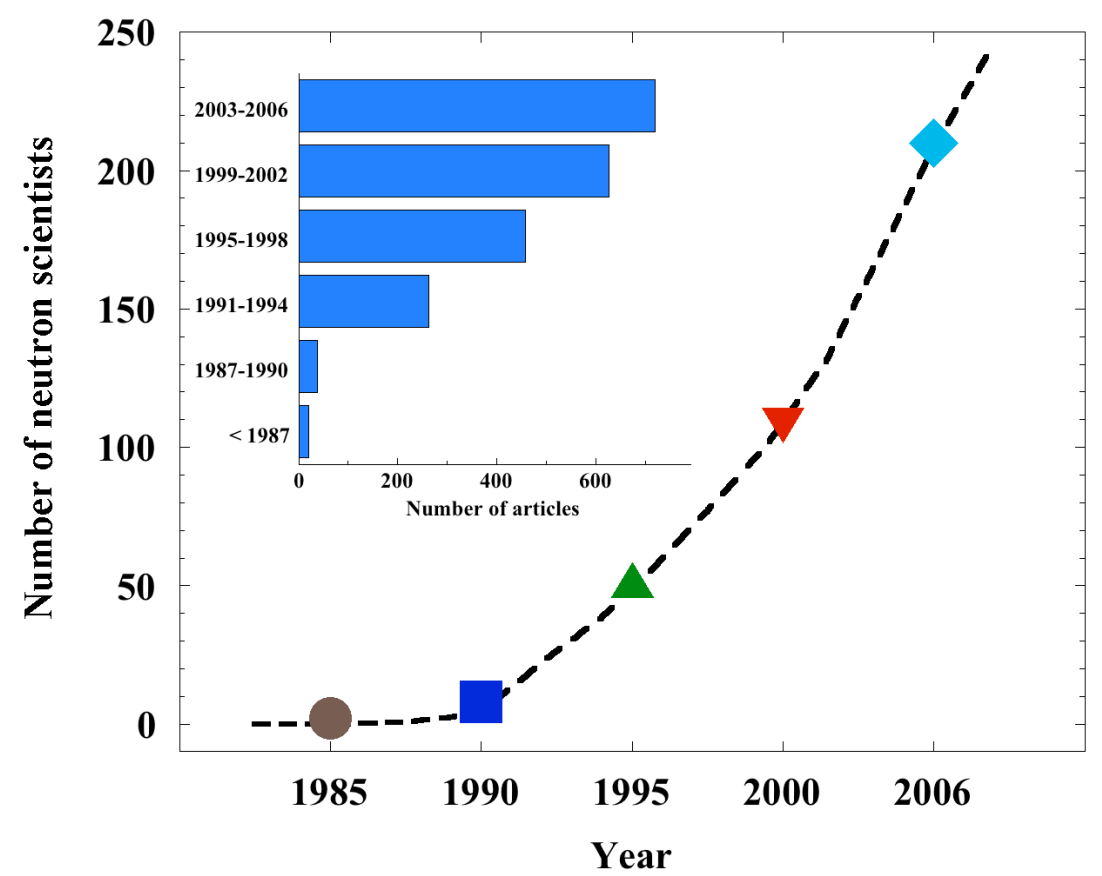

Figure 1: Increase in the number of Spanish "frequent users" of neutron scattering techniques each 5-year period (see text for details). Inset: Evolution of the number of articles published by Spanish scientists in 4-year periods. 